\title{
International clinical practice guidelines
}

\section{for early psychosis}

\section{INTERNATIONAL EARLY PSYCHOSIS ASSOCIATION WRITING GROUPI}

\begin{abstract}
Summary These international clinical practice guidelines were developed with detailed input from 29 invited international consultants, who provided content as well as detailed feedback on draft versions. The final draft of the guidelines was ratified by the Executive of the International Early Psychosis Association and presented and formally endorsed at the Third International Conference on Early Psychosis held in Copenhagen, September 2002. They have been revised slightly to include medications that were not available in 2002, although a fully comprehensive process of update has not yet been conducted. The final version is published in this Supplement with the aim of encouraging further discussion as well as providing practical guidance to clinicians and researchers. A second edition is planned for publication in 2008.
\end{abstract}

\section{INTRODUCTION}

The delivery of care in early psychosis is often delayed, piecemeal and alienating to patients and families. Patients usually have to cross a high threshold of disturbance and risk to gain access to treatment, and have to demonstrate a persistent and pervasive level of disability (or activity limitation/ participation restriction) to 'earn' the right to continuing care. Treatment models are geared to the needs of older patients with chronic conditions, reinforcing the pessimism inherent in the concept of schizophrenia. Community ignorance, stigma (operating in the larger community and within mental health services), poor mental health literacy, and the isolation of psychiatry from the rest of medicine and healthcare add to the obstacles to reforming systems to focus on early intervention. A special focus on the early phases of psychotic disorders is justified on three major grounds:

- The clinical care of people with firstepisode psychosis is often delayed or inadequate, and sometimes crude or harmful. Some people never receive treatment.

- Increasing evidence suggests there are major opportunities for effective secondary prevention, which could substantially lower the mortality and morbidity associated with these disorders.

- Epidemiological, neurobiological and psychosocial study of early psychosis may facilitate early intervention and secondary prevention, and enable superior treatment.

There are three targets for preventive interventions in early psychosis:

- The prepsychotic phase is often prolonged and characterised by subtle and confusing symptoms. Much of the disability associated with the psychotic disorders is established and accumulates in this phase.
- The period of untreated psychosis is a risk factor for a poor outcome. It has many determinants, but there is potential for intervention within communities to reduce the duration of untreated psychosis and the distress, risk and disability associated with untreated psychosis.

- The first psychotic episode and the critical period of the early years following initial diagnosis deserves optimal, comprehensive and phasespecific treatment with continuity of care guaranteed.

This draft consensus statement identifies key principles in addressing current deficiencies for preventive intervention and proposes strategies to enhance clinical care. The statement has been developed with input from the 29 invited international consultants who gave feedback by the publication deadline. The guidelines were presented and ratified at the Third International Conference on Early Psychosis held in Copenhagen in September 2002.

\section{GENER AL STATEMENTS}

- Early identification of people in the earliest phases of psychotic disorders combined with optimal treatment is likely to reduce the burden of disease. Early treatment of active psychosis is beneficial in its own right, but the possibility exists that it will also improve long-term outcomes and reduce the prevalence of psychotic disorders.

- Community-wide education should be encouraged to ensure that the public has a better understanding of the onset of psychotic disorders and how to obtain effective advice, treatment and support.

- Phase-specific programmes of care, which also take into account that the majority of patients with early psychosis are young, should be developed and evaluated.

- Pharmacological treatments should be introduced with great care in drugnaïve patients, with an overriding

I.The following international consultants contributed to the drafting of these guidelines which was endorsed at the third International Early Psychosis Conference, Copenhagen, September 2002:

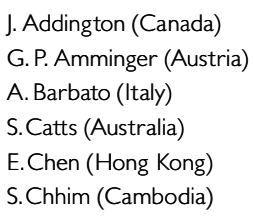

S. A. Chong (Singapore)
J. Cullberg (Sweden)
J. Edwards (Australia)
L. Grosso (France)
M. Louzã (Brazil)
M. Hambrecht (Germany)

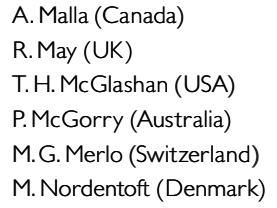

\author{
S. Nightingale (New Zealand) \\ D. Perkins (USA) \\ R. Thara (India) \\ K. Yamamoto (Japan) \\ A. Yung (Australia)
}


principle of doing the least harm while aiming for the maximum benefit. This will involve the use of the minimum effective dose of atypical or secondgeneration antipsychotics wherever possible, consistent with the recommendations of the World Psychiatric Association. If typical or first-generation antipsychotics cannot be avoided, which is the situation at present in developing countries, then they should be used judiciously and at very low doses.

- Psychosocial interventions have a fundamental place in early treatment, providing a humane basis for continuing care, preventing or resolving secondary consequences of the psychosis, and promoting recovery. Enhancement of professional skills and encouragement of psychosocial interventions by funding bodies are essential.

- Priority should be given to research on representative early psychosis populations to obtain a deeper understanding of the processes associated with the onset of psychosis.

- Consumers and families need to be engaged as partners in developing better treatments and with the aim of validating their experiences of early psychosis.

\section{PREPSYCHOTIC PERIOD}

\section{Background}

The prepsychotic period can be divided into two phases:

- The premorbid phase is the period during childhood and usually at least part of adolescence in which emotional, cognitive and behavioural functioning are not impaired.

- The prodromal period or symptomatic 'at-risk mental state' is usually characterised by a sustained and clinically important deviation from the premorbid level of experience and behaviour. The term 'at-risk mental state' refers to phenomenology and behaviour irrespective of the outcome.

\section{Clinical guidelines}

- The possibility of a psychotic disorder should be carefully considered in a young person who is becoming more socially withdrawn, performing worse for a sustained period at school or at work, or who is becoming more distressed or agitated yet unable to explain why.

- Some young people are at very high risk and meet the criteria for having an at-risk mental state. Criteria include:

- subthreshold positive symptoms that are not severe or persistent enough to meet criteria sufficient for a diagnosis of a DSM-IV/ICD10 psychotic disorder other than 'Brief Psychotic Disorder'; or

- a family history of psychotic disorder or schizotypal disorder in a first-degree relative plus a significant, persistent but non-specific decline in psychosocial functioning within the past year or so.

- If young people with an at-risk mental state are actively seeking help for the distress and disability associated with their symptoms, they need to be:

- engaged and assessed;

- offered regular monitoring of mental state and offered support;

- offered specific treatment for syndromes, such as depression, anxiety or substance misuse, and assistance with problem areas such as interpersonal, vocational and family stress if present;

- provided with psychoeducation and encouraged to develop coping skills for subthreshold psychotic symptoms;

- offered family education and support;

- provided information in a flexible, careful and clear way about risks for mental disorders as well as about existing syndromes.

- Such care can be carried out in a lowstigma environment, such as home, primary care or a youth-friendly officebased setting.

- Antipsychotic medications are not usually indicated unless the person meets criteria for a DSM-IV/ICD-10 psychotic disorder. Exceptions should be considered when rapid deterioration is occurring; severe suicidal risk is present and treatment of any depression has proved ineffective; or aggression or hostility are increasing and pose a risk to others. If antipsychotics are considered, ideally, atypical medications should be used in low doses and considered as a 'therapeutic trial' for a limited period. If there is benefit and resolution of symptoms after 6 weeks, the medication may be continued with the patient's consent for a further 6 months to 2 years, following explanation of risks and benefits. After this period, a gradual attempt to withdraw the medication should be made if the patient agrees and there has been a good recovery. If the patient has not responded to one atypical antipsychotic, another may be tried if the above indications still exist.

- If young people with an at-risk mental state are not seeking help, then regular contact with family members or friends may be an appropriate strategy.

- The evidence of effectiveness of treatments aimed specifically at reducing the risk of transition psychosis (e.g. cognitive and family therapy, antipsychotic medication or experimental neuroprotective drug strategies) remains preliminary. More data are required and the risk/benefit ratio of various interventions needs to be determined.

\section{Research}

- There should be better characterisation of the process of transition to prodrome or at-risk mental state to frank psychosis at phenomenological, psychosocial and neurobiological levels.

- Further research is needed to determine which treatment strategies may be effective in reducing the burden of symptoms and disability in at-risk mental states, and in reducing the risk of progression to a psychotic disorder.

- Such research must meet the highest ethical standards applicable to all medical research. Patients must give genuine informed consent and be free to withdraw from research at any time. Non-participation in research must not affect access to appropriate clinical care.

- Research should be led or heavily informed by local clinicians and researchers so that culturally normal experiences and behaviours are not misconstrued as pathological.

\section{FIRST EPISODE OF PSYCHOSIS}

\section{Clinical guidelines: access}

- The community should be well informed about psychotic disorders and how to obtain effective help. Community-wide initiatives to fight 
stigma associated with psychosis are required.

- Primary healthcare professionals should be competent in eliciting and recognising the early clinical features of psychotic disorder, just as they are expected to detect new cases of potentially serious and life-threatening physical illnesses, such as diabetes and hypertension.

- Enhanced undergraduate and postgraduate medical education and close links between primary and specialist mental health services are vital.

- Mental health services should provide user-friendly easy access to assessment and treatment for people who may be experiencing a first episode of psychosis. Assessment should be timely, depending on urgency, and flexible in terms of location.

- Ideally, treatment should commence prior to the development of a crisis, such as self-harm, violence or aggression, substance misuse and its consequences, or vocational failure. Early intervention has the potential to allow engagement outside these emotionally charged situations, providing a safer and more positive start to treatment. In-patient care and tranquillising medication are less likely to be needed if early intervention can be achieved.

- Families should be included in the assessment process and treatment plan, and this may be particularly relevant in developing countries.

\section{Clinical guidelines: location of treatment}

- Treatment should be provided in outpatient services or the home, if it is possible to give effective intervention in these settings, in order to minimise trauma, disruption and anxiety for the patient and the family.

- In-patient care may be required if there is a significant risk of self-harm or aggression, if the level of support in the community is insufficient, or if the degree of crisis is too great for the family to manage. A psychosocial element to treatment is essential to manage the crises that face patients and families who are attempting to cope with a disturbing situation.

- In-patient care should ideally be provided in units that provide services targeting early psychosis; the age and developmental stage of patients will also need to be taken into account.

- In-patient units should be small in size and adequately staffed so that nursing of highly distressed or agitated young people is possible without locking the unit. A secure area is necessary so that care can be provided for aggressive or manic patients without harming or disturbing other patients.

- When it is not possible to offer inpatient care in an early psychosis unit, a special section may be created in a general acute unit for young recentonset patients. In addition to the practical and social effects, this symbolically communicates that young people differ in both needs and prognosis from older patients with chronic conditions.

- Some settings provide acute day services and, if specialised early psychosis care is available, may be an appropriate alternative to in-patient admission.

- If attempts to engage the person in treatment fail and the person remains actively psychotic, in most cases the person has a right to be treated on an involuntary basis (depending on local mental health legislation).

\section{Clinical guidelines: initial management}

- Before initiating treatment, it is important to consider physical illnesses that can cause psychosis.

- Extrapyramidal side-effects from antipsychotic treatment should be avoided in order to encourage future adherence to medication. Although typical antipsychotics may be as efficacious as atypical antipsychotics in reducing positive psychotic symptoms, they are frequently less well tolerated even at low doses. For this reason alone, atypical antipsychotics should be used as first-line therapy, commencing with a low dose and titrating upwards very slowly over a period of several weeks ('start low, go slow').

- Examples of appropriate initial target doses for most patients are risperidone $2 \mathrm{mg} /$ day or olanzapine $7.5-10.0 \mathrm{mg} /$ day. Initial target doses of other medications such as quetiapine, ziprasidone and amisulpride are yet to be established. Half to two-thirds of patients might be expected to achieve a good response in positive psychotic symptoms within 3 weeks at the initial dose, but if necessary the doses can be increased to $4 \mathrm{mg} /$ day risperidone or $20 \mathrm{mg} / \mathrm{day}$ olanzapine. The level of clinical response and risk should be assessed frequently, but the dose of the antipsychotic should be increased only at widely spaced intervals (after initial titration, usually 14-21 days) if the response has been inadequate, and then only within the limits of sedation and the emergence of extrapyramidal sideeffects. However, extrapyramidal sideeffects should not be tolerated. If the response is not adequate at therapeutic doses by 6-8 weeks, another atypical antipsychotic should be tried. When use of typical antipsychotics is unavoidable, they should be commenced at very low doses (1-2 mg haloperidol or equivalent) and titrated very slowly within the limits of extrapyramidal side-effects. Generally, this will be a maximum of $4-6 \mathrm{mg}$ haloperidol or equivalent in first-episode psychosis.

- Low doses of antipsychotic medication will not have a rapid effect on distress, insomnia and behavioural disturbances secondary to psychosis; skilled nursing care, a safe and supportive environment, and regular and liberal doses of benzodiazepines are essential interim components of management in many cases. Although some atypical antipsychotics have initial sedative side-effects, treatment of psychosis should be separated conceptually from the need for tranquillisation.

- If positive psychotic symptoms persist after a trial of two first-line atypical antipsychotics (around 12 weeks), the reasons for the failure of treatment should be reviewed. Possible contributing factors include adherence problems, family stresses and substance misuse. Slow recovery of early treatment resistance of this kind is of concern and requires more intensive intervention.

- Clozapine and cognitive-behavioural therapy for persistent symptoms are obvious alternatives to consider.

- Supportive crisis plans are needed to facilitate recovery and acceptance of treatment. Specific psychosocial strategies should be employed when poor adherence, family stresses, increased suicide risk and substance misuse occur.

- Families are usually in crisis at the point of initiation of treatment and require emotional support and practical advice. 
- Families and other members of the person's social network, possibly including friends, teachers and employers, should be progressively informed and educated about the nature of the problem, treatments and the outcomes expected.

- If there are frequent relapses or slow recovery, a more intensive and prolonged psychoeducational and supportive intervention for families may be required. A calm and optimistic approach is vital, especially if the early course is stormy or there are additional family problems or secondary consequences of untreated psychosis.

- Family therapy may be indicated when there is a high degree of distress in the family.

- Structured group programmes tailored to the immediate needs of the patient should be available.

\section{RECOVERY (6-18 MONTHS) AND THE CRITICAL PERIOD (UP TO 5 YEARS)}

The time periods of 6-18 months for 'recovery' and up to 5 years as the 'critical period' are speculative. Time for remission of symptoms and risk of recurrence is highly variable and largely unknown.

\section{Background}

- It is essential that high-quality and intensive biopsychosocial care is provided continuously and assertively during the critical years after the onset of psychosis. This standard is rarely met, because patients are usually required to manifest negative sequelae, such as relapse, suicide attempts or severe disability, before care is provided in a reactive and 'too little, too late' manner.

- Attempts should be made to ensure continuity of care, with 'treating clinicians' remaining constant for at least the first 18 months of treatment.

- Relapses are common during the first few years after the onset of a psychotic disorder and the vulnerability to relapse persists in about $80 \%$ of patients.

- Consumers and families must be involved and empowered.

Correspondence: Professor Patrick McGorry, MD, PhD, FRCP, FRANZCP, Department of Psychiatry, University of Melbourne and ORYGEN Research Centre, Locked Bag 10/35, Poplar Road, Parkville, Victoria 3052,

Australia.

\section{Clinical guidelines}

- The impact of the prodromal phase and the psychosis itself on the person, on the family, and on developmental and vocational tasks should be determined. Strategies offered within a casemanagement model may include supportive psychotherapy, with an active problem-solving orientation, and negotiating occupational pursuits, including employment and/or education.

- Families should be provided with ongoing support and information, wherever possible in a partnership that involves the patient, family members and treating clinicians.

- Severe and potentially recurrent and disabling disorders of any kind in an adolescent or young adult can destabilise and distort the complex and often strained process of separation and individuation from the family. It is important to work within this context and not to misinterpret genuine attempts to cope.

- Psychological and psychosocial treatments should be core elements in the critical period and should be used to assist resolution of enduring positive and negative symptoms, the management of secondary comorbidity, and the promotion of recovery and positive mental health. Recovery work should emphasise the need to find meaning and develop mastery in relation to the psychotic experience.

- Multi-family groups (with or without the patient present) which have a psychoeducation focus should be provided.

- Depression, suicide risk, substance misuse and social anxiety in the patient should be identified and actively treated.

- Side-effects of antipsychotic medication, such as weight gain, sexual dysfunction and sedation, can retard recovery and should be monitored regularly. Once psychosis has achieved a sustained remission, slow reduction of antipsychotic medication should be tried, with the aim of determining the minimal dose required.
- A balance is needed between vigilance for early signs of relapse and 'space' to recover and resume the challenges of normal development.

- Antipsychotic medication reduces the risk of relapse in the early years after onset and, particularly when there is a diagnosis of schizophrenia, should be considered as an essential basis for sustained recovery. Relapse is distressing and may increase the risk of treatment resistance and other 'collateral damage', including worsening stigma.

- The optimal length of time that maintenance antipsychotic treatment is needed to minimise risk of relapse in firstepisode psychosis is not known. Strong recommendations cannot be made about the optimal duration of maintenance treatment, although current clinical practice varies from recommending continuation for 1 year after treatment initiation to indefinite duration of antipsychotic maintenance treatment. Against advice, many young patients wish to cease medication. Individuals who elect to cease medication (prematurely or otherwise) should continue to be monitored frequently and receive ongoing support. In all instances, careful psychoeducation about the risks and possible manifestations of relapse should be provided, accompanied by frequent review and support with unhindered access to early psychiatric treatment in the event of relapse.

- In the absence of clear evidence about when to cease antipsychotic medication it is pragmatic to take into account the severity of the first episode. For example, a fully remitted patient may be presented the option of gradually withdrawing medication after full remission for 12 months; if the first psychotic episode was severe and slow to respond, continuation of medication for a 2-year period following remission might be suggested. If the patient makes an incomplete recovery but has benefited significantly from medication, then it should be continued for at least 2-5 years. Long-term medication is advisable for individuals who experience frequent relapses. 
- Early warning signs of relapse should be discussed with the patient and family.

- If a patient who rejects treatment has persistent symptoms or experiences frequent relapses, with a pattern of high-risk, suicidal or aggressive behaviour, and is poorly engaged in treatment, then involuntary community treatment with or without depot medication may be required. This undesirable outcome should be considered to be time-limited to allow intervention and/or time to assist with acceptance of treatment recommendations. Involuntary and other restrictive treatment practices should be subject to frequent review and a planned termination date.
- Patients should remain in comprehensive, multidisciplinary, specialist mental healthcare throughout the early years of psychosis and, once their acute symptoms improve, not be discharged or transferred to primary care without continuing specialist involvement. However, partnerships can be established between a specialist centre, primary care and other agencies that can contribute to optimal care. First-episode psychosis is difficult to treat well, confers high levels of risk, and is the phase with the potential for greatest cost-effectiveness of treatment. To treat in a reactive manner is less effective and misses the best opportunity for enhancing outcomes and quality of life for patients and families.
- For a subgroup of patients (e.g. patients with personality issues and/or enduring positive symptoms) long-term psychotherapy may be indicated.

- Good relations between specialist early intervention services and general psychiatric services need to be fostered in order to facilitate the eventual transfer of care for a significant subgroup of individuals, because early intervention services are, by definition, time-limited.

- Consumers and families with recent experience of early psychosis should be encouraged to participate in the development and monitoring of early psychosis services. 